\title{
Correction to: longitudinal associations between built environment characteristics and changes in active commuting
}

\author{
Lin Yang ${ }^{1}$, Simon Griffin ${ }^{1,2}$, Kay-Tee Khaw ${ }^{2}$, Nick Wareham ${ }^{1}$ and Jenna Panter ${ }^{1 *}$
}

\section{Correction}

Following the publication of this article, Yang et al., [1] it has been brought to our attention that there were errors in the abstract and in Tables 2 and 3. These errors occurred as previous versions of the tables were uploaded to the submission system in the process of revising the paper. These errors relate to the presentation of the results. The results reported in the text of the paper are correct and the conclusions are unaltered. We also noted an error in the abstract.

\section{Error in Table 2}

Table 2 reports the characteristics of a sample. The correct version of Table 2 is presented below.

\section{Error in Table 3}

We also noted that the results presented in Table 3 are incorrect. The correct version of the table appears below and the results reported in the text match the results presented in this Table.

\section{Error in the abstract}

Those living in neighbourhoods with greater density of employment locations were more likely to maintain their active commuting.

\footnotetext{
Author details

${ }^{1}$ MRC Epidemiology Unit \& UKCRC Centre for Diet and Activity Research (CEDAR), University of Cambridge, School of Clinical Medicine, Box 285, Cambridge Biomedical Campus, Cambridge CB2 OQQ, UK. ²Department of Public Health and Primary Care, School of Clinical Medicine, Box 285, Cambridge Biomedical Campus, Cambridge CB2 OSR, UK.
}

Received: 9 October 2017 Accepted: 9 October 2017

Published online: 23 October 2017

\author{
Reference \\ 1. Yang L, Griffin S, Khaw KT, Wareham N, Panter J. Longitudinal associations \\ between built environment characteristics and changes in active \\ commuting. BMC Public Health. 2017;17:458. https://doi.org/10.1186/s12889- \\ 017-4396-3.
}

Correspondence: jrp63@medschl.cam.ac.uk

${ }^{1}$ MRC Epidemiology Unit \& UKCRC Centre for Diet and Activity Research

(CEDAR), University of Cambridge, School of Clinical Medicine, Box 285,

Cambridge Biomedical Campus, Cambridge CB2 0QQ, UK

Full list of author information is available at the end of the article 
Table 2 Descriptive characteristics of participants in the EPIC-

Norfolk cohort included in the analyses

\begin{tabular}{ll}
\hline Characteristic & $\%(n)$ \\
\hline Age (in years) & $30.4(839)$ \\
$\quad<50$ & $36.4(1003)$ \\
$50-54$ & $33.2(915)$ \\
$>=55$ & \\
BMl (kg/m2) & $43.4(1197)$ \\
Normal weight & $43.6(1203)$ \\
Overweight & $13.0(357)$ \\
Obese & \\
Social Class & $50.6(1395)$ \\
Professional & $36.1(996)$ \\
Skilled & $13.3(366)$ \\
Partly Skilled/unskilled & \\
Marital Status & $14.2(392)$ \\
Not married & $85.8(2365)$ \\
Married & \\
Alcohol Consumption & $6.1(170)$ \\
Non drinker & $80.1(2208)$ \\
Sensible drinker & $13.8(379)$ \\
Heavy drinker & \\
Smoking Status & $38.5(1062)$ \\
Never smoke & \\
Former smoker & \\
Current smoker & \\
\hline & \\
\hline &
\end{tabular}

Table 3 Adjusted associations between neighbourhood and route environment characteristics and uptake and maintenance of active commuting

\begin{tabular}{|c|c|c|c|c|}
\hline & \multicolumn{2}{|c|}{ Switching to active commuting $(n=2099)$} & \multicolumn{2}{|c|}{ Maintenance of active commuting $(n=658)$} \\
\hline & OR $(95 \% \mathrm{Cl})$ & $p$ & OR $(95 \% \mathrm{Cl})$ & $p$ \\
\hline \multicolumn{5}{|c|}{ Neighbourhood Environment } \\
\hline \multicolumn{5}{|c|}{ Density of employment locations (Reference: Lowest) } \\
\hline Second quartile & - & & $1.19(0.53,2.72)$ & 0.001 \\
\hline Third Quartile & - & & $1.53(0.70,3.36)$ & \\
\hline Highest & - & & $3.13(1.48,6.64)$ & \\
\hline \multicolumn{5}{|l|}{ Route Environment } \\
\hline \multicolumn{5}{|c|}{ Distance from home to work (Reference: <2 km) } \\
\hline $\mathrm{v} 2-10 \mathrm{~km}$ & $0.11(0.06,0.18)$ & 0.001 & $0.22(0.12,0.39)$ & 0.001 \\
\hline Over $10 \mathrm{~km}$ & $0.04(0.02,0.09)$ & & $0.06(0.25,0.13)$ & \\
\hline \multicolumn{5}{|c|}{ Main or secondary road on route (Reference: No) } \\
\hline Yes & $0.45(0.25,0.79)$ & 0.005 & $0.52(0.28,0.98)$ & 0.042 \\
\hline \multicolumn{5}{|c|}{ Number of streetlights per 100 m (Reference: Lowest) } \\
\hline Second quartile & $2.76(1.29,5.91)$ & 0.002 & - & \\
\hline Third Quartile & $2.02(0.94,4.34)$ & & - & \\
\hline Highest & $3.98(1.85,8.57)$ & & - & \\
\hline \multicolumn{5}{|c|}{ Total sample for analysis: $n=2757$} \\
\hline
\end{tabular}

